\title{
One Perspective: Regulatory Impact on Digital Imaging in the Electron Microscopy Laboratory
} Horn, Jeffrey W.*, Sterchi, Diane L.**

*Pathology Department, Toxicology Research Laboratories, Eli Lilly and Company, 2001 W. Main St, Greenfield, IN 46140

**Integrative Biology, Lilly Research Laboratories, Eli Lilly and Company, 2001 W. Main St, Greenfield, IN 46140

Electron microscopy (EM) digital image capture and image file storage has been gaining wide acceptance as a viable medium that is replacing or augmenting the negative film/chemical processing approach to data collection. In the bio-pharmaceutical electron microscopy laboratory the digital imaging changeover is a slower process due mainly to regulatory compliance and the complexities that surround '21 CFR Part 11 Electron Records; Electronic Signatures' (ER;ES)[1]. One perspective that will be presented addresses digital imaging compliance with federal guidelines and how an electron microscopy laboratory manager can organize documentation for compliance with ER;ES regulations.

Deeply rooted in the bio-pharmaceutical regulatory laboratory is the 'Code of Federal Regulations' (CFR) of Good Laboratory Practices (GLP) for procedural guidance on what needs to be performed and documented. Directions come from a written and approved set of Standard Operating Procedures (SOP) prepared by laboratory personnel and management for performing consistent and verifiable tasks. Standard operating procedures guide personnel to perform physical tasks and how to document, however, they do not verify the equipment software that generates or collects data. In essence, there are two distinct but inter-related sets of compliance issues (ER;ES and GLP's) related to assuring data integrity in a regulatory laboratory.

The 21 CFR Part 11, ER;ES was put into place by federal regulators for validating data collection from computer-based instrumentation. The 21 CFR Part 11, in short, states that "under certain circumstances, of electronic records, electronic signatures, and handwritten signatures executed to electronic records as equivalent to paper records and handwritten signatures executed on paper. These regulations, which apply to all FDA program areas, are intended to permit the widest possible use of electronic technology". This states it is imperative that any GLP facility using computer-based equipment and generating or storing electronic raw data is required to validate and qualify software programs.

Developing a qualified/validated system will depend on a company's computer system quality program, the needs of the EM lab, and their combined interpretation of the regulations. Bringing any digital imaging/digital capture software program to a validated status requires the EM laboratory personnel to address several issues, such as; the development plan, audit trail, legacy systems, use, security, system requirements, documentation, copies, and record retention. Validation is a process used to justify and document risk assessment, determine the potential of the system to affect product quality and safety, and record integrity. This process is documentation of impact on accuracy, reliability, integrity, availability, and authenticity of required records (images) and signatures. This process should also take into account the impact the systems have on your 
ability to meet predicate rule requirements and for meeting other regulatory mandates [2]. Therefore, an EM facility has several factors that must be documented in order to finalize the validation process.

The initial validation process requirements must address several major areas as specified in the ER;ES guidelines which are, but not limited to;

- Application source code, backup and restoration

- Business continuity plan

- Change control

- Disaster recovery plan

- Physical and logical security.

Furthermore, the system users must determine the program commands needed to capture images on a GLP study from any particular off-the-shelf program as part of the validation process. Once the facility determines which program commands are the most useful, a logical test plan is required. A logical test plan can consist of;

- Functionality

- Limitations

- Program problems

- Defect corrections [3].

To bring the whole package together once the complete validation package is approved, all applicable SOPs and training for end-users are required to be finalized prior to using the system. The SOPs for directing the end-user of the system should minimally encompass the system setup and installation, data collection and handling, system maintenance, data backup, recovery process, contingency plans, security, and change control $[2,3]$.

In conclusion, this work will hopefully provide the basic necessary information to organize, direct, and prepare an adequate approach to bring an EM laboratory digital image capture system into compliance for a regulatory audit. The changeover to digital imaging from negative film is an issue beyond the realm of this work but following this basic formula will help transition to regulatory compliance for using digital images.

[1] F.D.A. 21 CFR Part 11, Electronic Records; Electronic Signatures; Final Rule. Federal Register, 62(54), 13429 (March 20, 1997).

[2] D. Sterchi, National Society for Histotechnology Symposium/Convention (Sept 2004), Toronto, Canada, Workshop \#34.

[3] K.L. Keatley, Quality Assurance, 7 (1999) 77. 